\title{
Quantities, amounts, and the numerical core system
}

\author{
Avishai Henik ${ }^{\text {* }}$, Tali Leibovich ${ }^{2}$, Sharon Naparstek ${ }^{1}$, Liana Diesendruck ${ }^{3}$ and Orly Rubinsten ${ }^{4}$ \\ 'Department of Psychology, Zlotowski Center for Neuroscience, Ben-Gurion University of the Negev, Beer-Sheva, Israel \\ ${ }^{2}$ Department of Cognitive Sciences, Ben-Gurion University of the Negev, Beer-Sheva, Israel \\ ${ }^{3}$ Department of Computer Sciences, Ben-Gurion University of the Negev, Beer-Sheva, Israel \\ ${ }^{4}$ Department of Learning Disabilities and Edmond J. Safra Brain Research Center for the Study of Learning Disabilities, University of Haifa, Haifa, Israel \\ ${ }^{*}$ Correspondence: henik@bgu.ac.il
}

Numerical cognition is essential to many aspects of life and arithmetic abilities predict academic achievements better than reading (Estrada et al., 2004). Accordingly, it is important to understand the building blocks of numerical cognition, the neural tissue involved, and the developmental trajectories. In the last two decades research has made impressive strides forward in studying numerical cognition and brain mechanisms involved in arithmetic. This advance was marked by suggestions of a numerical core system that can be characterized as a set of intuitions for quantities innately available to humans (Brannon et al., 2006) and animals (Cantlon and Brannon, 2007). We suggest that another system, evolved to perceive and evaluate non-countable dimensions like size or amount of substance may be important for the evolution of the numerical system and numerical abilities. The current opinion article examines this idea and the possible interplay between, on the one hand perception and evaluation of continuous dimensions and, on the other, the numerical system.

\section{NEUROCOGNITIVE BASIS FOR OUANTIFICATION}

Several behavioral effects have been well documented in the field of numerical cognition. Amongst them are: subitizing, counting, and the distance effect. The first and second refer to the processes involved when participants are asked to enumerate - report the number of items in an array. Here, performance is best described as a biphasic graph: their reaction time (RT) rises slowly, between 40 and $100 \mathrm{~ms}$ per item, up to four items, and then rises steeply at a rate of $250-350 \mathrm{~ms}$ per item (Jevons, 1871; Trick and Pylyshyn, 1994). It seems that four items (or less) can be grasped almost simultaneously with no effort; this is termed subitizing (Kaufman et al., 1949). In contrast, for five to nine items, participants are engaged in an effortful counting process. The third behavioral effect, the distance effect, arises when participants are asked to compare two arrays of dots, or two numerals. RT decreases with increase in the distance between the to-be-compared stimuli. This numerical distance effect was first reported by Moyer and Landauer (1967) who suggested that people convert written or auditory numbers into analog magnitudes. The effect has been reported by many other researchers under various conditions (Banks et al., 1982; Dehaene, 1989; Link, 1990; Tzelgov et al., 1992; Schwarz and Heinze, 1998). Further research showed these effects (e.g., subitizing, counting, and distance) can be found in infants and animals. For example, the numerical distance effect has been found in children (Sekuler and Mierkiewicz, 1977; Mussolin and Noël, 2007; Holloway and Ansari, 2008; Landerl and Kölle, 2009), and in primates (Brannon, 2003; Cantlon and Brannon, 2006). In addition, the various effects are compromised in developmental dyscalculia (DD) or mathematical learning disability (MLD) (for subitizing and counting: Koontz and Berch, 1996; Geary et al., 1999; Landerl et al., 2004; Moeller et al., 2009, for the distance effect: Price et al., 2007; Rousselle and Noël, 2007; Mussolin et al., 2010). Finally, many reports have suggested involvement of the parietal lobes and in particular the intraparietal sulcus (IPS) in numerical cognition (Dehaene et al., 2003; Fias et al., 2003; Ansari et al., 2006; Cohen Kadosh et al., 2007a).

This accumulated body of results led to a widely accepted view of an innate domainspecific foundation for arithmetic. In particular, it has been claimed that there is a core numerical system, the basis of which is the ability to perceive and manipulate discrete quantities (e.g., enumeration of dots) (Ansari, 2008; Dehaene, 2009; Butterworth, 2010; Piazza, 2010). In addition, it has been conjectured that arithmetic disability involves a domain-specific deficit in the capacity to enumerate (Butterworth, 2010;
Piazza, 2010). However, careful scrutiny of the literature suggests that to achieve a comprehensive picture of numerical cognition other factors need to be examined.

\section{CAREFUL EXAMINATION OF CORE QUANTIFICATION}

A survey of the literature raises several interesting observations; (1) similar to their sensitivity to discrete quantities (e.g., enumeration of dots), infants show sensitivity to non-countable continuous dimensions like area (Brannon et al., 2006), line length (de Hevia and Spelke, 2010), and size (Lourenco and Longo, 2010). Mix et al. (2002) surveyed the literature on quantification in infancy and early childhood and suggested that the literature provides no clear-cut evidence that infants use numbers to perform quantitative tasks. Moreover, they summarized that there is evidence that infants respond to amount of substance, rather than discrete numbers, in what had seemed to be numerical tasks. (2) Developmental trajectories similar to those with numbers (i.e., the size of the distance effect has been found to decrease over developmental time Sekuler and Mierkiewicz, 1977) have been shown in other areas (see example for the Stroop effect reported by Schiller, 1966) and seem to rely on domaingeneral rather than domain-specific abilities (Holloway and Ansari, 2008). During development, language may provide a medium that bridges between core domain-specific systems (Munkholm, 2001; Spelke, 2003; Platt and Spelke, 2009). Last but not least, (3) numbers are intimately associated with non-countable dimensions (e.g., size, brightness). Numerical values and physical sizes interfere with one another - the size congruity effect (Henik and Tzelgov, 1982; Tzelgov et al., 1992; Cohen Kadosh et al., 2008c) and the same apply to the relationship between numbers and brightness (Cohen Kadosh and Henik, 2006). Importantly, Lourenco and Longo (2010) 
reported a size congruity effect in infants that were 9 months old. They presented convincing evidence for an intimate relationship between numerosity and size; infants who learned to expect an association between color and size of objects also expected to see a similar association between color and numerosity. Moreover, the size congruity effect is compromised in DD (Rubinsten and Henik, 2005, 2006).

This short review of the literature suggests that other factors might contribute to the development of the number sense. In particular, it is possible that the need and ability to evaluate non-countable dimensions (e.g., sizes or amounts) helped to develop or to improve the domain-specific "core knowledge" of arithmetic, which extracts numerosity of sets (Dehaene, 2009).

\section{SIZES, AMOUNTS, AND THE NUMERICAL SYSTEM}

The size congruity effect is dependent on numerical proficiency. Irrelevant numerical values will not affect relevant physical-size judgments unless the participant is proficient with the numerical system. Consequently, it was found that the size congruity effect develops with age (Rubinsten et al., 2002). In recent years this effect was employed to study proficiency with the number system and automaticity (i.e., processing even when not part of a task requirement) in numerical processing (Rubinsten et al., 2002; Cohen Kadosh et al., 2007a; Szucs and Soltesz, 2007). Recent research indicates that it involves the IPS: It was found to be deficient in a patient who suffered from a brain injury in the IPS (Ashkenazi et al., 2008) and imaging studies (fMRI) have shown that it is correlated with IPS activation (Cohen Kadosh et al., 2007a, 2008b; Szucs and Soltesz, 2007). Interestingly, other aspects of objects like brightness (Cohen Kadosh et al., 2008a), ordinal position, and time also involved the IPS (Simon et al., 2002; Walsh, 2003; Cantlon et al., 2009).

Those with DD show deficient processing in size congruity (Rubinsten and Henik, $2005,2006)$ and temporary lesioning of the IPS by transcranial magnetic stimulation (TMS) produce a DD-like pattern of the size congruity effect (Cohen Kadosh et al., 2007b). Importantly, DD subjects show deficiency not only in size congruity but also in other dimensions that interact with numbers like brightness and height (Rubinsten and Henik, 2005). Hence, it is conceivable that the basis of DD or MLD or its precursor lies with a difficulty in processing and evaluation of sizes or amounts. In a recent article, Bugden and Ansari (2011) examined the relationship between the size congruity effect and math performance in first and second grade children. They reported that size congruity did not predict math performance. This is not in line with our expectations. However, this might be due to the restriction of the range of the predictor (i.e., size congruity) or math performance or both. Alternatively, it is possible that size congruity might predict performance in older children because of its dependence on math proficiency.

Imaging data as well as behavioral results led to suggestions that "...countable and uncountable quantity...should be represented with the same kind of symbols (mental magnitudes)... to determine behaviorally important decisions" (Gelman and Gallistel, 2000), and that important computational demands of an action system (reaching, grasping) are the basis for the involvement of the parietal lobes in comparative judgment tasks (Walsh, 2003). Namely, it has been suggested that the parietal lobes reflect computational demands of the brain dorsal system involved in perception for action (Goodale et al., 1991; Goodale, 2000). However, Cantlon et al. (2009) have recently suggested that the ability to evaluate magnitudes (e.g., size) might underlie the development of the numerical system. This is in line with the general view suggested in the current proposal. Namely, the evolution of the dorsal brain system might have been dependent on the ability to compute size and size differences. A neurocognitive system that handles this aspect of cognition (evaluation of size or amount) might have been instrumental for the development of the occipito-parietal dorsal brain system (perception for action). This same system (evaluation of size or amount) helped develop or improve the numerical system.

Interestingly, recent works on perception of objects using various size and distance illusions suggested that perceived rather than retinal size modulates activation of early visual areas (i.e., v1; Murray et al., 2006; Sterzer and Rees, 2006; Fang et al., 2008). Moreover, according to Goldfarb and Tzelgov (2005), perceiving size, on the basis of monocular distance cues, is automatic and modulates numerical processing. It is possible that size is processed very early both in terms of visual feature extraction and in terms of timing in the visual stream. This information is fed forward to the parietal lobes (and other brain structures) to serve other systems and goals (e.g., the dorsal brain system).

In a recent article, Piazza (2010) reviewed two early pre-verbal systems: the approximate number system (ANS) and the object tracking system (OTS). She suggests that children are equipped with both systems before symbolic learning takes place, each system is based on dedicated neural circuits, and each undergoes a separate developmental trajectory. Piazza concludes that the ANS rather than the OTS is crucial for the development of numerical cognition. The ANS represents numbers in an approximate fashion. However, the ANS (and OTS) involves enumeration of discrete quantities and obeys Weber's law. The current proposal focuses on the ability to evaluate and perceive continuous and non-countable properties. Needless to say, evaluation of continuous variables is approximate by definition. Similarly, Gebuis and Gevers (2011) recently suggested that continuous visual properties, like area subtended by dot arrays, modulate performance that was earlier suggested to rely on numerosity.

\section{A PRECURSOR OF THE NUMERICAL SYSTEM?}

We suggest that routines and neural structures built for size judgments were made available to other systems (e.g., language), through evolution, due to the need to develop an exact numerical system. We have focused on the existence of noncountable representations and the ability to perceive and evaluate sizes or amounts. We suggest that the ability to perceive and evaluate sizes might be a more primitive system that was exploited, throughout the years, as the basis for the development of the number sense and numerical abilities. Whether this system was "...hi-jacked to perform judgments along a new dimension (e.g., number)" (Cantlon et al., 2009), was shaped by cultural needs (Dehaene, 2005; Dehaene and Cohen, 2007), or became accessible to the numerical system through evolution (Rozin, 1976) is not clear. But 
this system might be the antecedent of the numerical system. Note that this fits in with the idea put forward by Piaget that continuous spatial extent serves as the antecedent to a child's concept of discrete numbers (Feigenson, 2007). Going back to the discussion of the OTS (Piazza, 2010), it seems that object individuation is a necessary process on the way to a discrete numerical system. Within the framework of the current proposal, it is important to find out how organisms moved from evaluation and perception of continuous non-countable properties to perception and enumeration of individual objects, or more generally, how these two abilities affected one another.

Interestingly, the visual system is characterized by a similar arrangement. Humans have two co-existing visual pathways. In addition to the primary, evolutionarily younger, geniculo-striate system there exists an evolutionarily older system - the retino-tectal pathway. These two systems have somewhat different roles but are connected and have mutual effects (Henik et al., 1994). The evolutionarily older system deals mainly with spatial aspects of vision and connects to the parietal lobes. Interestingly, the evolutionarily older system is critical in blind-sight when the primary visual system is damaged (Rafal et al., 1990). As alluded to earlier, it is quite possible that the number and the size/amount systems evolved to coexist, similar to these two visual systems.

Alternatively, it is possible that evaluation of size/amount is not based on a separate system but rather only one system exists. This could be a system that processes both numerosity and sizes or amounts. Both aspects of this system evolved together due to changing natural requirements. Namely, moving between the requirement to develop an enumeration ability under certain circumstances and the requirement to evaluate sizes (note Helmholtz's unconscious inference, which explains size constancy) under other conditions. A situation like this would create a modular system whose modules are used interchangeably (Kashtan and Alon, 2005).

In order to gain a comprehensive picture of numerical cognition, there is a need to study the ability to perceive and evaluate sizes and amounts and their relationship to the development of the numerical system.

\section{REFERENCES}

Ansari, D. (2008). Effects of development and enculturation on number representation in the brain. Nat. Rev. Neurosci. 9, 278-291.

Ansari, D., Dhital, B., and Siong, S. C. (2006). Parametric effects of numerical distance on the intraparietal sulcus during passive viewing of rapid numerosity changes. Brain Res. 1067, 181-188.

Ashkenazi, S., Henik, A., Ifergane, G., and Shelef, I. (2008). Basic numerical processing in left intraparietal sulcus (IPS) acalculia. Cortex 44, 439-448.

Banks, W. P., Mermelstein, R., and Yu, K. H. (1982). Discrimination among perceptual and symbolic stimuli. Mem. Cognit. 10, 265-275.

Brannon, E. M. (2003). Number knows no bounds. Trends Cogn. Sci. (Regul. Ed.) 7, 279-281.

Brannon, E. M., Lutz, D., and Cordes, S. (2006). The development of area discrimination and its implications for number representation in infancy. Dev. Sci. 9, F59-F64.

Bugden, S., and Ansari, D. (2011). Individual differences in children's mathematical competence are related to the intentional but not automatic processing of Arabic numerals. Cognition 118, 32-44.

Butterworth, B. (2010). Foundational numerical capacities and the origins of dyscalculia. Trends Cogn. Sci. (Regul. Ed.) 14, 534-541.

Cantlon, J. F., and Brannon, E. M. (2006). Shared system for ordering small and large numbers in monkeys and humans. Psychol. Sci. 17, 401-406.

Cantlon, J. F., and Brannon, E. M. (2007). How much does number matter to a monkey? J. Exp. Psychol. Anim. Behav. Process. 33, 32-41.

Cantlon, J. F., Platt, M. L., and Brannon, E. M. (2009). Beyond the number domain. Trends Cogn. Sci. (Regul. Ed.) 13, 83-91.

Cohen Kadosh, R., Cohen Kadosh, K., and Henik, A. (2008a). When brightness counts: the neuronal correlate of numerical-luminance interference. Cereb. Cortex 18, 337-343.

Cohen Kadosh, R., Cohen Kadosh, K., Henik, A., and Linden, D. E. J. (2008b). Processing conflicting information: facilitation, interference, and functional connectivity. Neuropsychologia 46, 2872-2879.

Cohen Kadosh, R., Henik, A., and Rubinsten, O. (2008c). Are Arabic and verbal numbers processed in different ways? J. Exp. Psychol. Learn. Mem. Cogn. 34, 1377-1391.

Cohen Kadosh, R., Cohen Kadosh, K., Linden, D. E. J., Gevers, W., Berger, A., and Henik, A. (2007a). The brain locus of interaction between number and size: a combined functional magnetic resonance imaging and event-related potential study. J. Cogn. Neurosci. 19, 957-970.

Cohen Kadosh, R., Cohen Kadosh, K., Schuhmann, T., Kaas, A., Goebel, R., Henik, A., and Sack, A. T. (2007b). Virtual dyscalculia induced by parietal-lobe TMS impairs automatic magnitude processing. Curr. Biol. 17, 689-693.

Cohen Kadosh, R., and Henik, A. (2006). A common representation for semantic and physical properties: a cognitive-anatomical approach. Exp. Psychol. 53, 87-94.

de Hevia, M. D., and Spelke, E. S. (2010). Numberspace mapping in human infants. Psychol. Sci. 21, 653-660.

Dehaene, S. (1989). The psychophysics of numerical comparison: a reexamination of apparently incompatible data. Percept. Psychophys. 45, 557-566.
Dehaene, S. (2005). "Evolution of human cortical circuits for reading and arithmetic: the neuronal recycling hypothesis," in From Monkey Brain to Human Brain, eds J. R. D. S. Dehaene, M. Hauser, and G. Rizzolatti (Cambridge, MA: MIT Press), 133-157.

Dehaene, S. (2009). Origins of mathematical intuitions: the case of arithmetic. Ann. N. Y. Acad. Sci. 1156, 232-259.

Dehaene, S., and Cohen, L. (2007). Cultural recycling of cortical maps. Neuron 56, 384-398.

Dehaene, S., Piazza, M., Pinel, P., and Cohen, L. (2003). Three parietal circuits for number processing. Cogn. Neuropsychol. 20, 487-506.

Estrada, C.A., Martin-Hryniewicz, M., Peek, B. T., Collins, C., and Byrd, J. C. (2004). Literacy and numeracy skills and anticoagulation control. Am. J. Med. Sci. 328, 88-93.

Fang, F., Boyaci, H., Kersten, D., and Murray, S. O. (2008). Attention-dependent representation of a size illusion in human V1. Curr. Biol. 18, 1707-1712.

Feigenson, L. (2007). The equality of quantity. Trends Cogn. Sci. (Regul. Ed.) 11, 185-187.

Fias, W., Lammertyn, J., Reynvoet, B., Dupont, P., and Orban, G. A. (2003). Parietal representation of symbolic and nonsymbolic magnitude. J. Cogn. Neurosci. $15,1-11$.

Geary, D. C., Hoard, M. K., and Hamson, C. O. (1999). Numerical and arithmetical cognition: patterns of functions and deficits in children at risk for a mathematical disability. J. Exp. Child Psychol. 74, 213-239.

Gebuis, T., and Gevers, W. (2011). Numerosities and space; indeed a cognitive illusion! A reply to de Hevia and Spelke (2009). Cognition 121, 248-252.

Gelman, R., and Gallistel, C. R. (2000). Non-verbal numerical cognition: from reals to integers. Trends Cogn. Sci. (Regul. Ed.) 4, 59-65.

Goldfarb, L., and Tzelgov, J. (2005). Is size perception based on monocular distance cues computed automatically? Psychon. Bull. Rev. 12, 751-754.

Goodale, M. A. (2000). "Perception and action in the human visual system," in The New Cognitive Neurosciences, ed. M. Gazzaniga (Cambridge, MA: MIT Press), 365-377.

Goodale, M. A., Milner, A. D., Jakobson, L. S., and Carey, D. P. (1991). A neurological dissociation between perceiving objects and grasping them. Nature 349 , 154-156.

Henik, A., Rafal, R., and Rhodes, D. (1994).Endogenously generated and visually guided saccades after lesions of the human frontal eye fields. J. Cogn. Neurosci. 6, 400-411.

Henik, A., and Tzelgov, J. (1982). Is three greater than five: the relation between physical and semantic size in comparison tasks. Mem. Cognit. 10, 389-395.

Holloway, I. D., and Ansari, D. (2008). Domain-specific and domain-general changes in children's development of number comparison. Dev. Sci. 11, 644-649.

Jevons, W.S. (1871). The power of numerical discrimination. Nature 3, 281-282.

Kashtan, N., and Alon, U. (2005). Spontaneous evolution of modularity and network motifs. Proc. Natl. Acad. Sci. U.S.A. 102, 13773-13778.

Kaufman, E. L., Lord, M. W., Reese, T. W., and Volkmann, J. (1949). The discrimination of visual number. Am. J. Psychol. 62, 498-525.

Koontz, K. L., and Berch, D. B. (1996). Identifying simple numerical stimuli: processing inefficiencies exhibited by arithmetic learning disabled children. Math. Cogn. $2,1-23$. 
Landerl, K., Bevan, A., and Butterworth, B. (2004). Developmental dyscalculia and basic numerical capacities: a study of 8-9-year-old students. Cognition 93, 99-125.

Landerl, K., and Kölle, C. (2009). Typical and atypical development of basic numerical skills in elementary school. J. Exp. Child Psychol. 103, 546-565.

Link, S. (1990). Modeling imageless thought: the relative judgment theory of numerical comparison. J. Math. Psychol. 34, 2-41.

Lourenco, S. F., and Longo, M. R. (2010). General magnitude representation in human infants. Psychol. Sci. 21, 873-881.

Mix, K. S., Huttenlocher, J., and Levine, S. C. (2002). Multiple cues for quantification in infancy: is number one of them? Psychol. Bull. 128, 278-294.

Moeller, K., Neuburger, S., Kaufmann, L., Landerl, K., and Nuerk, H.-C. (2009). Basic number processing deficits in developmental dyscalculia: evidence from eye-tracking. Cogn. Dev. 24, 371-386.

Moyer, R. S., and Landauer, T. K. (1967). Time required for judgement of numerical inequality. Nature 215, 1519-1520.

Munkholm, P. (2001). Language, space, and the development of cognitive flexibility in humans: the case of two spatial memory tasks. Cognition 79, 263-299.

Murray, S. O., Boyaci, H., and Kersten, D. (2006). The representation of perceived angular size in human primary visual cortex. Nat. Neurosci. 9, 429-434.

Mussolin, C., Mejias, S., and Noël, M.-P. (2010). Symbolic and nonsymbolic number comparison in children with and without dyscalculia. Cognition 115, 10-25.

Mussolin, C., and Noël, M.-P. (2007). The nonintentional processing of Arabic numbers in children. J. Clin. Exp. Neuropsychol. 29, 225-234.

Piazza, M. (2010). Neurocognitive start-up tools for symbolic number representations. Trends Cogn. Sci. (Regul. Ed.) 14, 542-551.
Platt, M. L., and Spelke, E. S. (2009). What can developmental and comparative cognitive neuroscience tell us about the adult human brain? Curr. Opin. Neurobiol. 19, 1-5.

Price, G. R., Holloway, I., Räsänen, P., Vesterinen, M., and Ansari, D. (2007). Impaired parietal magnitude processing in developmental dyscalculia. Curr. Biol. 17, 1042-1043.

Rafal, R., Smith, J., Krantz, J., Cohen, A., and Brennan, C. (1990). Extrageniculate vision in hemianopic humans: saccade inhibition by signals in the blind field. Science 250, 118-121.

Rousselle, L., and Noël, M.-P. (2007). Basic numerical skills in children with mathematics learning disabilities: a comparison of symbolic vs non-symbolic number magnitude processing. Cognition 102, 361-395.

Rozin, P. (1976). "The evolution of intelligence and access to the cognitive unconscious," in Progress in Psychobiology and Physiological Psychology, eds J. M. Sprague and A. N. Epstein (New York, NY: Academic Press), 245-280.

Rubinsten, O., and Henik, A. (2005). Automatic activation of internal magnitudes: a study of developmental dyscalculia. Neuropsychology 19, 641-648.

Rubinsten, O., and Henik, A. (2006). Double dissociation of functions in developmental dyslexia and dyscalculia. J. Educ. Psychol. 98, 854-867.

Rubinsten, O., Henik, A., Berger, A., and Shahar-Shalev, S. (2002). The development of internal representations of magnitude and their association with Arabic numerals. J. Exp. Child Psychol. 81, 74-92.

Schiller, P.H. (1966). Developmental study of color-word interference. J. Exp. Psychol. 72, 105-108.

Schwarz, W., and Heinze, H.-J. (1998). On the interaction of numerical and size information in digit comparison: a behavioral and event-related potential study. Neuropsychologia 36, 1167-1179.

Sekuler, R., and Mierkiewicz, D. (1977). Children's judgement of numerical inequality. Child Dev. 48, 630-633.
Simon, O., Mangin, J.-F., Cohen, L., Le Bihan, D., and Dehaene, S. (2002). Topographical layout of hand, eye, calculation, and language-related areas in the human parietal lobe. Neuron 33, 475-487.

Spelke, E. (2003). "What makes us smart? Core knowledge and natural language," in Language in Mind: Advances in the Investigation of Language and Thought, eds D. Gentner and S. Goldin-Meadow (Cambridge, MA: MIT Press), 277-311.

Sterzer, P., and Rees, G. (2006). Perceived size matters. Trends Cogn. Sci. (Regul. Ed.) 9, 302-304.

Szucs, D., and Soltesz, F. (2007). Event-related potentials dissociate facilitation and interference effects in the numerical Stroop paradigm. Neuropsychologia 45, 3190-3202.

Trick, L. M., and Pylyshyn, Z. W. (1994). Why are small and large numbers enumerated differently? A limited capacity preattentive stage in vision. Psychol. Rev. 101, 80-102.

Tzelgov, J., Meyer, J., and Henik, A. (1992). Automatic and intentional processing of numerical information. J. Exp. Psychol. Learn. Mem. Cogn. 18, 166-179.

Walsh, V. (2003). A theory of magnitude: common cortical metrics of time, space and quantity. Trends Cogn. Sci. (Regul. Ed.) 7, 483-488.

Received: 03 September 2011; accepted: 22 December 2011; published online: 20 January 2012.

Citation: Henik A, Leibovich T, Naparstek S, Diesendruck $L$ and Rubinsten $O$ (2012) Quantities, amounts, and the numerical core system. Front. Hum. Neurosci. 5:186. doi: 10.3389/fnhum.2011.00186

Copyright (C) 2012 Henik, Leibovich, Naparstek, Diesendruck and Rubinsten. This is an open-access article distributed under the terms of the Creative Commons Attribution Non Commercial License, which permits non-commercial use, distribution, and reproduction in other forums, provided the original authors and source are credited. 